\title{
MULTI-DIMENSIONAL EVALUATION OF THE PRESENCE OF SARCOPENIA IN PATIENTS WITH KNEE OSTEOARTHRITIS
}

\author{
Sefa Aslan ${ }^{1}$ and Hakan Genc ${ }^{2}$ \\ ${ }^{1}$ Ankara Gaziler Physical Therapy and Rehabilitation Training and Research Hospital \\ ${ }^{2}$ Ankara Training and Research Hospital
}

April 22, 2021

\begin{abstract}
Aim: In this study, we evaluated the presence of sarcopenia multidimensionally in patients with knee osteoarthritis (OA) using clinical, ultrasonographic and biochemical parameters, and in this respect, it was aimed to investigate the relation between OA and sarcopenia and to identify the most practical, easily accessible and inexpensive method for investigating sarcopenia.Materials and methods: 102 patients with clinical and radiological diagnosis of knee osteoarthritis and 33 healthy control subjects were included in the study. The detailed musculoskeletal system examination of the patients included in the study was carried out by a single physician, and blood sample tests were recorded .Dual-X-ray absorptiometry (DEXA) is used to measure Body composition parameters and muscle mass measurements, isometric muscle strength evaluations, handgrip strength and gait speeds for diagnosis of sarcopenia. Short-form -36 (SF-36) The Nutritional Assessment-short form (MNA), the Western Ontario and McMaster Universities Osteoarthritis Index (WOMAC), the International Physical Assessment Questionnaire Short Form (IPAQ-SF) and the Center for Epidemiologic Studies Depression Scale (CES-D scale) were administered to every patient as outcome measures.Results: Body composition parameter results showed that sarcopenic patients had statistically lower values as fat mass, lean body mass and skeletal muscle index ( $\mathrm{p}<0.001, \mathrm{p}=0.001, \mathrm{p}<0.001$, respectively) than those of non-sarcopenic and control group. It was determined that body composition values measured with DEXA, ultrasonographic measures, isokinetic muscle strength assessment, handgrip strength and gait speed had predictive values for sarcopenia. Conclusion: We found that patients with sarcopenic OA were older, weaker, undernourished, and restricted in their level of physical activity in the study in which we identified sarcopenia as approximately $12 \%$ in patients with osteoarthritis. Among the methods of determining sarcopenia, ultrasound becomes prominent with its practical, cheap and easily accessible features. We think that our results will increase the awareness of the presence of sarcopenia in OA patients.
\end{abstract}

\section{Multi-Dimensional Evaluation Of The Presence Of Sarcopenia In Patients With Knee Os- teoarthritis}

Sefa GÜMRÜK ASLAN ${ }^{a}$, MD, Hakan GENÇ ${ }^{\mathrm{b}}$, MD

a University of Health Sciences, Gaziler Physical Medicine and Rehabilitation Training and Research Hospital, Department of Physical Medicine and Rehabilitation, Ankara, Turkey

b University of Health Sciences, Ankara Training and Research Hospital, Department of Physical Medicine and Rehabilitation, Ankara, Turkey

\section{ABSTRACT}

Aim: In this study, we evaluated the presence of sarcopenia multidimensionally in patients with knee osteoarthritis (OA) using clinical, ultrasonographic and biochemical parameters, and in this respect, it was aimed 
to investigate the relation between OA and sarcopenia and to identify the most practical, easily accessible and inexpensive method for investigating sarcopenia.

Materials and methods : 102 patients with clinical and radiological diagnosis of knee osteoarthritis and 33 healthy control subjects were included in the study. A total of 135 subjects were evaluated using the European Working Group on Sarcopenia in Older People (EWGSOP) diagnostic criteria of sarcopenia. The first group consists of $(\mathrm{OA})$ patients with sarcopenia, the second group consist of OA patients without sarcopenia and the third group is controls subjects. The detailed musculoskeletal system examination of the patients included in the study was carried out by a single physician, and complete blood count, renal function tests, electrolytes, total protein, albumin, $25(\mathrm{OH})$ vitamin $\mathrm{D}$, serum leptin, serum adiponectin, PTH, TSH and vitamin B12 values were recorded using the venous blood samples taken from the study group.Dual-X-ray absorptiometry (DEXA) is used to measure Body composition parameters and muscle mass measurements, isometric muscle strength evaluations, handgrip strength and gait speeds for diagnosis of sarcopenia. Short-form -36 (SF-36) The Nutritional Assessment-short form (MNA), the Western Ontario and McMaster Universities Osteoarthritis Index (WOMAC), the International Physical Assessment Questionnaire Short Form (IPAQ-SF) and the Center for Epidemiologic Studies Depression Scale (CES-D scale) were administered to every patient as outcome measures.

Results: The mean age of the group with sarcopenia was statistically higher than the other two groups ( $\mathrm{p}<0.001)$. The weight, body mass index (BMI), waist circumference, upper mid-arm circumference, thigh and leg circumference of osteoarthritis (OA) patients with sarcopenia were statistically lower than those of non-sarcopenic and control group $(\mathrm{p}<0.01-\mathrm{p}<0.001)$. Body composition parameter results showed that sarcopenic patients had statistically lower values as fat mass, lean body mass and skeletal muscle index ( $\mathrm{p}<0.001, \mathrm{p}=0.001, \mathrm{p}<0.001$, respectively) than those of non-sarcopenic and control group and fat mass index values $(\mathrm{p}=0.012)$ are lower than the non-sarcopenic group. With respect to sarcopenia, the effect of adiponectin and leptin levels were not detected. It was determined that body composition values measured with DEXA, ultrasonographic measures, isokinetic muscle strength assessment, handgrip strength and gait speed had predictive values for sarcopenia.

Conclusion: We found that patients with sarcopenic OA were older, weaker, undernourished, and restricted in their level of physical activity in the study in which we identified sarcopenia as approximately $12 \%$ in patients with osteoarthritis. Among the methods of determining sarcopenia, ultrasound becomes prominent with its practical, cheap and easily accessible features. We think that our results will increase the awareness of the presence of sarcopenia in OA patients.

Keywords: Sarcopenia, osteoarthritis, ultrasound, Dual-X-ray absorptiometry, body composition, isokinetic evaluation, leptin, adiponectin

Trial registration: Registry: ClinicalTrials.gov, Registration number: NCT04828200. Date of registration: 1 April 2021. Retrospectively registered.

\section{WHAT'S KNOWN?}

-Sarcopenia and OA have been postulated to be co-existing conditions. The relationship between sarcopenia and OA is still unclear and no strong consensus has been reached.

- Sarcopenia is often associated with frailty, falls, fractures and disability.

\section{WHAT'S NEW?}

- The presence of sarcopenia in patients with knee OA is evaluated multidimensional way, including body composition parameters, ultrasonographic muscle structure evaluations, isometric muscle strength evaluations, handgrip strength and walking speed evaluations, biochemical evaluations, anthropometric measurements and comprehensive functional evaluation tests measured by DEXA were examined.

- The relation between OA and sarcopenia was aimed to be investigated as well as the most practical, easily accessible and inexpensive method for investigating sarcopenia was aimed to be identified. 


\section{INTRODUCTION}

Osteoarthritis $(\mathrm{OA})$ is one of the most common joint disorders worldwide. The knee is the most common symptomatic joint in osteoarthritis. In epidemiological studies conducted in various regions of the world, it has been reported that $10-30 \%$ of people over the age of 65 have symptomatic knee OA $(1,2)$.

The elderly population is increasing in the world and constitutes the majority of the population, especially in developed countries. As people age, they are more likely to suffer from the problems. The term sarcopenia was first used by Irwin Rosenberg to describe the loss of muscle mass associated with ageing (3). Today sarcopenia is defined as a syndrome characterized by the general and progressive loss of skeletal muscle mass and strength. Sarcopenia contributes to several adverse health outcomes, including loss of mobility and independence, reduced quality of life, fall and fracture risk, frailty, and mortality $(3,4)$. Many factors contribute to the development of sarcopenia. These include the aging process itself, deficiencies in the optimal diet, immobility / sedentary life, chronic diseases, and the use of many medications (3).

It has been found that the development of sarcopenia is faster in patients with OA, and this has been found to be associated with increased inflammatory cytokines in OA (5). TNF- $\alpha$ and IL-1 $\beta$ play an important role in the increase in protein catabolism, which explains the muscle loss that leads to a decrease in physical activity in individuals with joint pain $(6,7)$. In this study, the presence of sarcopenia in patients with knee $\mathrm{OA}$ is evaluated multidimensionally using clinical, ultrasonographic and biochemical parameters. In this respect, the relation between $\mathrm{OA}$ and sarcopenia was aimed to be investigated as well as the most practical, easily accessible and inexpensive method for investigating sarcopenia was aimed to be identified.

\section{PATIENTS AND METHODS}

A total of 102 patients ages 50 to 70 years who were admitted to University of Health Sciences, Ankara Training and Research Hospital, Physical Medicine and Rehabilitation Department between April 2016 and September 2016, were diagnosed with knee osteoarthritis according to the American College of Rheumatology criteria (8) and 33 healthy volunteers (mean age 54,6 years; range, 52 to 69 years) were enrolled in this study.A total of 135 subjects were evaluated by the European Working Group on Sarcopenia in Older People (EWGSOP) diagnostic criteria (4) for the diagnosis of sarcopenia. The first group consists of knee OA patients with sarcopenia, the second group consist of OA patients without sarcopenia and the third group is the control group, which is not being exposed to be sarcopenia or knee OA. In addition, the patients who have had knee replacement surgery on the affected limb or for whom outcomes will be measured after knee replacement surgery or who have a concomitant joint disease such as rheumatoid arthritis or gout will be excluded because these conditions or procedures will confound the assessment of the outcomes of the interventions and those with a history of malignant disease, hyperthyroidism/ hypothyroidism, chronic inflammatory disease, diabetes mellitus, and uncontrollable heart and kidney diseases and pregnant women were excluded. The detailed musculoskeletal system examination of the patients included in this study was carried out by a single physician, and whole blood count, C-reactive protein, erythrocyte sedimentation rate, and routine biochemistry tests were performed using the venous blood samples taken from the study group. Written informed consent was obtained from each participant. The study protocol was approved by the Ethics Committee of University of Health Sciences, Ankara Training and Research Hospital, (16/03/2016/633). The study was conducted in accordance with the principles of the Declaration of Helsinki.

\section{ASSESSMENT TOOLS}

After obtaining written informed consent, the participants'demographic details were noted and were screened for the following risk factors of sarcopenia: history of smoking, quality of life using Short Form-36 (SF-36) (9), malnutrition using Mini-Nutritional Assessment (MNA-SF) (10), physical inactivity using the International Assessment Questionnaire Short Form (IPAQ-SF) (11), history of depression using The Center for Epidemiologic Studies Depression Scale (CES-D scale) (12) and the timed 'Up \& Go' test using which scale were used to assess functional performance (13).

Complete blood count, renal function tests, electrolytes, total protein, albumin, $25(\mathrm{OH})$ vitamin $\mathrm{D}$, serum 
leptin, serum adiponectin, PTH, TSH and vitamin B12 values were recorded. Anthropometric measurements such as waist, hip, upper-middle arm (MAC), calf circumference (CC) and thigh and subcutaneous fat measurements from 3 regions (triceps, biceps, thigh) were measured with the caliper and recorded. Quadriceps-hamstring Peak Torque (PT) values and the ratio of PT values to body weight (PT / VA) (at 60 and $180^{\circ} /$ sec speeds) were evaluated with an isokinetic dynamometer. The Western Ontario and McMaster Universities Arthritis Index (WOMAC) were used in the evaluation of knee Osteoarthritis (14). The radiological staging was evaluated with the Kellgren Lawrence (KL) scale (15).

Sarcopenia was diagnosed according to the sarcopenia diagnosis criteria published by EWSGOP using walking speed, hand grip strength, anthropometric measurements and body composition parameters which were determined by DEXA method. The muscle masses of the patients were evaluated by measuring the fat mass (Fat Mass-FM), fat mass index (FMI), lean body mass (LBM) and skeletal muscle index (SMI) calculated by DEXA. Appendicular muscle mass (ASM) is calculated by summing the lean soft tissue of the two upper limbs and the two lower limbs. Skeletal muscle mass index is calculated by dividing the appendicular lean mass by the square of the neck height [SMI = ASM / height2 $(\mathrm{kg} / \mathrm{m} 2)]$. Those with both low muscle mass and muscle strength were considered sarcopenic. All ultrasonographic examinations were performed by a single physiatrist experienced in musculoskeletal sonography. All ultrasonographic measurements were taken by the same physician experienced in musculoskeletal sonography in order to avoid interindividual variability. Ultrasound measurements was performed in B-mode using 5-12 MHz linear multifrequent transducer (Logic e portable; GE Healthcare, China). The transducer was placed perpendicularly to the long axis of the muscles with adequate use of contact gel and minimal pressure to avoid excessive compression of the muscles. Each examination was performed bilaterally while patients were in supine and prone position. Rectus femoris muscle thickness was assessed on the bilateral side of the patient, in a supine position with both knees extended and relaxed and toes pointing to the ceiling, at the halfway point between epicondylus lateralis and trochanter major of the femur. For the evaluation of the rectus abdominal muscle the probe was positioned longitudinally to the muscle, brain-caudal direction. Two images were placed, one to two centimeters to the right and one to two centimeters to the left of the umbilicus. Gastrocnemius medialis (GM) muscle thickness was assessed at the medial part of the GM muscle belly: half of the distance from the popliteal crease to the center of the medial malleolus. All A set of three consecutive measurements was performed, and the average value was reported as muscles thickness. Data were reported in centimeters $(\mathrm{cm})$ as means \pm standard deviation. The thicknesses of subcutaneous were measured in the axial view at the same point. Additionally, fascicle length and pennation angle were measured between the two parallel aponeuroses of the gastrocnemius muscle (bulkiest part of medial head) in the longitudinal view when subjects were in prone position with their ankles at $90^{\circ}$. Comprehensive evaluation tests, anthropometric measurements and laboratory parameters, initially measured muscle ultrasound parameters, were compared in patients with and without sarcopenia. All measurements and blood samples for the control group were measured in the same way.

\section{STATISTICAL ANALYSIS}

Statistical analysis was performed using the IBM SPSS version 23.0 software (IBM Corp., Armonk, NY, USA). Descriptive data were expressed in mean \pm standard deviation (SD) for normally distributed variables, median and 'minimum-maximum' values for non-normally distributed variables. After looking at the statistical difference with the Mann-Whitney U test in two group comparisons and the Kruskal Wallis test in more than two group comparisons, Dunn-Bonferroni double comparison test was performed. The relationship between the numerical variables was examined using the Spearman correlation coefficient. The strength of the relationship was determined according to the correlation coefficient ( $\mathrm{r}$ ). A $\mathrm{r}$ value of 0 to 0.2 was considered very weak, 0.2 to 0.4 weak, 0.4 to 0.6 moderate-to-severe, 0.6 to 0.8 strong, and 0.8 to 1 very strong relationship. A p-value of $<0.05$ was considered statistically significant. A multiple lineer regression model was used to identify predictors sarcopenia. The model fit was assessed using appropriate residual and goodness-of-fit statistics. A $5 \%$ type -I error level was used to infer statistical significance. The criteria value to consider the variables for the multivariate analyses was $\mathrm{p}<0.10$. Standardized coefficients ( $\beta$, ranging from -1 to 1 ) for all independent variables included in the regression equations, coefficient of 
determination(r2) and correlation coefficient $(\mathrm{r})$ were analyzed for all...independent variables.Statistical significance was set at $\mathrm{p}<0.05$.

\section{RESULTS}

Within the scope of the study, a total of 152 individuals; 117 patients who were admitted to the physical therapy and rehabilitation outpatient clinic with knee pain who were also diagnosed with knee osteoarthritis, and 35 healthy individuals as control group were examined. Fifteen OA patients and two of kontrol individuals were excluded. A total of 135 individuals were evaluated with the EWGSOP diagnostic criteria for the presence of sarcopenia. Twelve (11.8\%) of 102 patients with osteoarthritis were sarcopenic. There were no sarcopenic individuals in the control group. OA patients with sarcopenia constituted the first group(Group 1), non-sarcopenic OA patients constituted the second group (Group 2), and controls healty individuals constituted the third group (Group 3). Distribution of some descriptive characateristics among study groups are presented in Table 1. The weight, BMI, waist circumference, hip circumference, upper-middle arm circumference, thigh and leg circumference of patients group 1 were statistically lower than the group 2 and group 3. ( $\mathrm{p}<0.01-\mathrm{p} 0.001)$. In the non-sarcopenic osteoarthritis group, weight and height of the patients were lower than the control group. Biceps, triceps, and thigh extensor surface measurements were found to be less in the group with sarcopenia than in the other groups $(\mathrm{p}<0.001-\mathrm{p}=0.002)$. Bilateral hand grip strength and gait speed of sarcopenic patients were found to be statistically lower than non-sarcopenic and control groups. All of the patients group 1 were at a low level in the physical activity assessment questionnaire. Thirty eight of the patients with group 2 were at a low level, 44 were at a moderate level, 8 were at a very active level. Twenty one individuals of the group 3 were at a low level, 9 were at a moderate level and 3 were found to be doing very active physical activity. The metabolic equivalents (METs) values obtained from IPAQ-SF were also calculated as min/week (Table 2). The METs values were found to be statisticall lower in the group 1 compared to the group $2(\mathrm{p}=0.017)$. The mean MNA score was found to be statistically significantly lower in the group 1 compared to the other two groups $(\mathrm{p}<0.001)$ (Table 2). In the CES-D evaluation, the score was calculated as $25.58 \pm 11.73$ (5-52) in the sarcopenic group, $22.47 \pm 14.07$ (4-54) in the group 2 and $15.15 \pm 10.85(0-49)$ in the group 3 (Table 2 ).

When OA groups with and without sarcopenia were evaluated with the WOMAC score and the WOMAC pain and stiffness values were compared, it was found that there was no significant difference between the sarcopenic and non-sarcopenic osteoarthritis groups $(\mathrm{p}>0.05)$. When the WOMAC physical function scores were compared, a significant difference was found between the groups $(\mathrm{p}<0.05)$. The WOMAC total scores were statistically higher in the sarcopenic group than in the non-sarcopenic osteoarthritis group $(\mathrm{p}=0.006)$.

The pain that was experienced by patients at night, on the move and at rest were compared with VAS and no significant difference between groups were found No statistically significant difference was found between the group1 and 2 in the calculated sub-parameters of SF-36 used to evaluate the quality of life ( $\mathrm{p}>0.05)$.

When laboratory parameters were evaluated, calcium levels of patients group 1 were statistically lower than group $2(\mathrm{p}=0.023)$, leptin $(\mathrm{p}=0.720)$, adiponectin $(\mathrm{p}=0.068)$ and vitamin $\mathrm{D}(\mathrm{p}=0.285)$ levels were not detected as statistically significant. In the comparison between the sarcopenic patients and the control group, vitamin D levels were found to be statistically significantly higher in the control group $(\mathrm{p}=0.030)$. Albumin levels in the control group $(\mathrm{p}=0.035)$ were found to be lower than the other two groups (Table 3).

When body composition parameters measured by DEXA, were compared in patients with and without sarcopenic OA and the control group, sarcopenic patients had statistically significantly lower fat masses, lean dry weights and SMI values ( $\mathrm{p}<0.001, \mathrm{p}=0.001$, $\mathrm{p} 0.001$, respectively), where as sarcopenic patients' FMI values $(\mathrm{p}=0.012)$ were found to be lower in patients than group2 and 3 . In the group 2 , it was found that the fat percentage and LBMI values $(\mathrm{p}<0.001, \mathrm{p}=0.002)$ were higher than the other two groups (Table 4).

When the study groups were compared in terms of muscle strength measured by the isokinetic dynamometer, average knee extension forces at $60^{\circ} / \mathrm{sec}$ angular velocity for the right and left knee were lower in the 
sarcopenic group when evaluated in terms of peak torque values $(\mathrm{p}<0.001, \mathrm{p} 0.001$, and the ratio of peak torque to body weight $(\mathrm{p}=0.007$ and $\mathrm{p}=0.031$, respectively). In the group 1 , right knee flexion forces at 180 degrees angular velocities and peak torque values were statistically significantly lower $(\mathrm{p}=0.038)$ than group 2 and 3.. Bilateral gastrocnemius medius fascicle lengths and right gastrocnemius medius pennate angles of sarcopenic patients were found to be statistically significantly lower than non-sarcopenic and control groups. Right gastrocnemius medius muscle thickness was significantly decreased in the sarcopenic group compared to the non-sarcopenic group $(\mathrm{p}=0.014)$. Left gastrocnemius medius muscle thickness was higher and rectus abdominis muscle thickness was statistically significantly lower in the non-sarcopenic group compared to the other study groups $(\mathrm{p}=0.025$ and $\mathrm{p}=0.001$. It was determined that there was no significant difference between the bilateral rectus femoris subcutaneous tissue thickness and muscle thickness between the groups $(\mathrm{p}>0.05)$

\section{(Table 5).}

No statistically significant correlation was found between muscle ultrasound evaluations and adiponectin and leptin levels within the sarcopenic group. It was found that there was also a weak positive correlation between adiponectin level and rectus abdominis muscle thickness within the non-sarcopenic group. A moderate-weak and positive correlation was found between the leptin level and bilateral gastrocnemius subcutaneous tissue thickness, left gastrocnemius muscle thickness, bilateral rectus femoris subcutaneous tissue thickness and rectus abdominis subcutaneous tissue thickness. When the relationship between body composition values measured by DEXA and muscle parameters measured by the US were examined; a statistically significant, moderate and positive correlation was found between FMI and rectus abdominis muscle thickness in patients of group 1. A statistically significant, strong and positive correlation was found between SMI and bilateral gastrocnemius muscle thickness.

When stepwise multiple linear regression analysis was performed, hip circumferences and gastrocnemius muscle thickness (Model 1) were found to be significant predictors for SMI ( $\mathrm{r}=0.998, \mathrm{r} 2=0.995)$. The significant variables ( $\mathrm{p}<0.05$ according to correlation analyses) included in Model 1 are age, body mass index, waist and hip circumferences, gait speed, rectus femoris and gastrocnemius medialis muscle thicknesses. Model 2 was included age, body mass index, waist and hip circumferences, gait speed, grip strength, rectus femoris and gastrocnemius medialis muscle thicknessesIn (Model 2); hip circumferences and gastrocnemius muscle thickness were significant predictors for gait speed $(\mathrm{r}=1.000, \mathrm{r} 2=1.000)$. Gastrocnemius muscle thickness was the strongest predictor (with a high standardized regression coefficient) for gait speed in both models.

There was a moderate positive correlation (r: 0.643, p: 0.024) between walking speed and vitamin D levels and a moderately negative correlation ( $\mathrm{r}:-0.615$, p: 0.033 ) with fat mass (FM). A moderate negative correlation (r:-0.594, p: 0.042) was found between the timed up to go test and leptin levels.

\section{DISCUSSION}

In this multidimensional study which investigated the presence of sarcopenia in patients with osteoarthritis, the rate of sarcopenia in knee OA patients was found to be approximately $12 \%$. Sarcopenic OA patients were found to be older, weaker, thinner, malnourished, and had limited physical activity levels. Unlike other studies, the presence of sarcopenia in a multidimensional way, including body composition parameters, ultrasonographic muscle structure evaluations, isometric muscle strength evaluations, handgrip strength and walking speed evaluations, biochemical evaluations, anthropometric measurements and comprehensive functional evaluation tests measured by DEXA were examined. As far as we know, this is the first study in the literature to use all of these parameters at the same time. In this study, all parameters except biochemical parameters were found to have predictive value in diagnosing sarcopenia.

Kemmler et al. in their study with elderly individuals over the age of 70 , found the prevalence of sarcopenia to be $4.5 \%$ and reported that patients with hip and lower extremity OA were more likely to have sarcopenia than those without osteoarthritis (9.1\% with OA; $3.5 \%$ without OA) (16). The rate of sarcopenia found in our study appears to be similar to Kemmler et al. Clinical evidence suggests that the link between OA and 
sarcopenia occurs through functional and cellular pathways. It has been reported that patients with OA and rheumatoid arthritis are more prone to the development of sarcopenia due to the increased cytokines (5).

Baumgartner et al., for the diagnosis of sarcopenia, used the appendicular muscle mass (ASM) and skeletal muscle mass index (SMI) calculated by DEXA and reported the prevalence of sarcopenia as 13-24\% under the age of 70 and $>50 \%$ in those aged 80 and over. Studies have found a strong correlation between DEXA and body composition measurements in elderly individuals with MR and CT measurements, which are gold standard methods (17).

In recent years, it has been suggested that muscle ultrasonography can be used as a practical, easily accessible and inexpensive method in the evaluation of sarcopenia. In the literature reviews, 4 studies were found in which DEXA and US were used together to diagnose sarcopenia. In all of these studies, ultrasonographic measurements of muscle thickness from many regions and thighs were found to be consistent with muscle mass values measured by DEXA.(18-21). In our study, the relationship between left gastrocnemius pennation angle and isokinetic muscle strength (peak torque extensor at an isokinetic $180^{\circ}$ angular velocity) and appendicular lean body mass measured by DEXA was shown. The relationship between the angle of pennation of the right gastrocnemius muscle were found to be correlated with muscle strength (peak torque at an isokinetic 180degree angular velocity and peak torque/body weight extensor ). A strong positive correlation was found between SMI and bilateral gastrocnemius medialis muscle thickness also.

The advantage of using DEXA in the diagnosis of sarcopenia is the better evaluation of SMI and skeletal muscle mass which was found to be associated with physical disability (22). Handgrip strength and quadriceps muscle strength are associated independently with predicting disability (23). Isometric handgrip strength, lower extremity muscle strength was found to be strongly correlated with knee extension torque and thigh cross-sectional muscle area (24). In our study, bilateral extensor isokinetic 60 degrees angular velocities of the knee joint were found to be statistically lower in the sarcopenic group compared to the other 2 groups. In functional evaluations, bilateral handgrip strength was found to be decreased in the sarcopenic group. Bilateral handgrip strength and bilateral knee extension strengths were positively correlated as moderate and with respect to several health outcomes.

Additionally, in this study, sarcopenia was found to be significantly associated with physical dependence, regardless of age, ethnic difference, comorbidities, health-related behaviours and fat mass (25). Gait speed plays an important role in the evaluation of sarcopenia. Gait speed is the first functional assessment to be considered in the evaluation of sarcopenia (26).

Active muscle strength test in the elderly is difficult to perform due to the reasons such as immobilization, pain, injury, surgical procedure, cognitive impairment, and technical complexity. The cross-sectional area of the muscles shows muscle mass and is related to muscle strength. Therefore, the cross-sectional area can be evaluated in order to have an idea about muscle strength in clinical applications. It has been stated that in intensive care patients, muscle loss can be monitored with rectus femoris and vastus intermedius muscle thickness (27). Although the quadriceps femoris pennation angle was not found to be significantly correlated with functional muscle parameters, in the study conducted by Strasser et al. with 26 healthy elderly individuals, the pennation angle was found to be lower than the control group consisting of 26 healthy young individuals (27). In our study, bilateral measurements were including in 3 regions by ultrasonograph. Fascicular length and pennation angle of the gastrocnemius muscle were evaluated as well as muscle thickness, and non-sarcopenic patients and healthy volunteers were included in the study as well as sarcopenic patients. In our study, it was found that bilateral gastrocnemius fascicle lengths and right-sided gastrocnemius pennate angle were lower in sarcopenic patients than those without and in the control group, and there was no significant difference between bilateral rectus femoris subcutaneous tissue and muscle thickness.

Decreased muscle quality and muscle mass can be considered as the most important factors in OA pathogenesis (28). At the same time, the painful joint can cause a decrease in muscle tone and consequently a decrease in muscle mass. When the relationship between OA and sarcopenia was examined, it could not be understood whether OA is a risk factor or a direct result of sarcopenia. For this, it may be recommended 
to conduct a systematic study investigating the development of sarcopenia in the ipsilateral extremity with OA occurring in a single joint and its effect on physical functions (29). In our study, the WOMAC function and total score evaluated in the sarcopenia group were found to be statistically significantly higher than the other two groups. The decrease in muscle mass and muscle quality caused by sarcopenia in patients with OA may explain the increasingng in WOMAC scores. In addition, a negative correlation between WOMAC function and total score and left-hand grip strength was found as an indicator of upper extremity muscle function in our function studies. This suggests that sarcopenia may affect not only the lower extremity but the whole body in these patients.

In our study, a relationship between rectus abdominis subcutaneous tissue thickness and right-hand grip strength, and between rectus abdominis muscle thickness and FMI was found. Although Abe et al. did not find a correlation between BMI and body mass with abdominal muscle thickness $(2-3 \mathrm{~cm}$ from the side of the umbilicus, lower part of the rectus abdominis) measured by ultrasound, a correlation with handgrip strength and standing up test performance was found. The body mass with the appendicular lean soft tissue mass (ALM) parameter measured by DEXA was evaluated (30). In another study using BIA in patients with metabolic syndrome, a positive correlation was found between rectus abdominis muscle thickness and skeletal muscle mass index (SMI) (31). In a cross-sectional study conducted by Kara et al. with 30 healthy volunteers, low vitamin D levels were found to be associated with decreased muscle strength (especially knee and ankle extensors), but no relationship was found with muscle architecture (32). In our study, a positive correlation was found between bilateral gastrocnemius medius muscle thickness and SMI and vitamin D levels. Therefore, it can be thought that Vitamin D supplementation may increase muscle mass and decrease sarcopenia in these patients.

In the study conducted by Kositsawat et al. where 1826 people were studied; a relationship was found between low vitamin D levels and the decreased walking speed (33). In our study, a negative relationship was found between gait speed, and FM measured by DEXA, and a positive relationship with vitamin D level. Although there was no significant difference between vitamin D levels in our study, the average vitamin D levels in the sarcopenic group were determined to be $19 \mathrm{ng} / \mathrm{mL}$. These levels can be considered as a deficiency in the literature and may explain the relationship between vitamin D levels and walking speed in this study. Additionally, in our study, a cross-sectional relationship between increased BMI index and decreased walking speed and a positive correlation between increased leptin levels and BMI was found. In another study, although no relationship was found between the thigh muscle cross-sectional area and the decrease in walking speed, a relationship was found between the change in the thigh muscle area and the change in walking speed. The total amount of fat and muscle fat infiltration has been reported to be important predictors for a reduction in walking speed (34). This report may likewise explain our findings.

As shown in our study, it is an expected result that sarcopenic patients are weaker (low body weight and BMI) and their bilateral hand grip strength, walking speed, waist and hip circumference, upper-middle arm circumference, calf and thigh circumferences are lower compared to non-sarcopenic patients. Similar to our findings, in a study conducted by Marini et al. with 207 sarcopenic people between the ages of 65-93, it was found that waist circumference, upper middle arm and calf circumference, weight and BMIs were significantly lower in both genders compared to those without sarcopenia (35).

The relation of sarcopenia and malnutrition has been shown in many studies $(36,37)$. In their study Velazquez et al. which involved 90 elderly women with an average age of 78 , it was found that the prevalence of sarcopenia was $41 \%$, when the nutritional status was evaluated according to MNA founded that $7.1 \%$ of those with normal, $45.9 \%$ of those with malnutrition risk, and $77.2 \%$ of those with nutritional deficiency were sarcopenic (38). In our study, MNA was used for malnutrition assessment and it was found that sarcopenic patients were significantly malnourished compared to non-sarcopenic and control groups. Therefore, it is our understanding that improving the nutritional status of OA patients may be beneficial in increasing muscle mass and preventing sarcopenia in these patients.

Leptin has been found to be increased in the cartilages of patients with OA, and although it is mainly synthesized from adipocytes, it is also synthesized from chondrocytes (18). A positive correlation has been 
found between the leptin level measured in synovial fluid and BMI, and it has been stated that this may be one of the metabolic factors explaining the role of obesity in the pathogenesis of OA (5). The function of leptin in OA is not fully determined but it has a biphasic effect which facilitates cartilage synthesis at low levels and causes inflammation and degeneration of cartilage at high levels (39). In our study, the relationship between sarcopenia, plasma leptin and adiponectin levels were evaluated and the relationship between leptin and adiponectin levels and muscle ultrasound and isokinetic evaluations of the patients were studied. A weak and low positive correlation was found between leptin levels and subcutaneous tissue thickness of the gastrocnemius and rectus femoris muscle in the muscle ultrasound evaluation performed in the non-sarcopenic knee OA group. This link may be due to the correlation between leptin and obesity.

In our study, although the serum leptin level was found to be lower in the sarcopenia group compared to the group without sarcopenia, there was no statistically significant difference between the two groups. Moreover, there were significantly different BMIs between these two groups. The BMI values in the sarcopenic group were significantly lower than the group 2 and 3 The lower serum leptin levels in the sarcopenia group compared to the group without sarcopenia may be related to lower BMI. The studies of Toussirot et al. and Sarl et al. stated that the low leptin levels found in patients with ankylosing spondylitis could be attributed to the low BMI values of the patient group in furtherance to our study $(40,41)$. In our study, the FM value measured by DEXA in sarcopenic patients was found to be lower than the other two groups. The low level of leptin in the sarcopenic group compared to the other two groups may be related to the low FM value.

Adiponectin plays a role in many processes such as energy metabolism, insulin resistance, and vascular physiology. High molecular weight adiponectin is the most active form of adiponectin which is the most sensitive marker reflecting insulin resistance and metabolic rate (42). High adiponectin levels have been recognized as a predictor for bone mineral density loss (43). In addition, high adiponectin levels have been shown to contribute to physical disability in a prospective cohort in which the effect of adiponectin on physical functions was investigated (44). In our study, a negative correlation was found between adiponectin levels and the VAS score in motion. This relationship was found in the sarcopenic group. The fact that adiponectin is seen as more determinant in terms of pain intensity in patients with low BMI, especially biomechanical factors, may be related to its role in inflammatory pathology. In our study, the relationship between adiponectin level and quality of life were also studied. In our study, the quality of life of the patients was evaluated using the SF-36 scoring system. When SF-36 sub-scores were compared with adiponectin levels in the OA group, a moderate negative correlation was observed between the pain sub-score. Quality of life can be affected by conditions such as disease activation, pain status, and the presence of comorbidities. It is a predictable situation that a more serious disease state will progress when quality of life worsens.

One of the limitations of our study is that the limit cut-off value for DEXA measurement was used according to the values calculated in previous studies. Since there is no muscle mass limit value determined for Turkish society, our evaluations was based on the studies with the highest number of patients and the values suggested by EWSGOP. In our study, only female patients, thinking that they would create a more homogeneous group, were included. Most previous studies investigating sarcopenia in OA patients also included only women in literature.

\section{CONCLUSIONS:}

In our study, where the rate of sarcopenia was found to be approximately $12 \%$ in patients with osteoarthritis, it is found that sarcopenic OA patients were older, weaker, less powerful, undernourished and limited in physical activity..Although the relationship between sarcopenia and OA cannot be fully explained, clinicians should be aware of the risk of developing sarcopenia in patients with OA, guide their patients to increase their physical activity, and provide nutrition and vitamin D support to increase their muscle mass.

It will be an important step to have low cost and easily accesible sarcopenia diagnostic with relatively simple methods. Our study is important in terms of showing the changes in the muscular structures of sarcopenic patients and showing that muscle ultrasound can be considered as a cheaper and faster method than other gold standard methods in the diagnosis of sarcopenia. However, this view should be supported by 
further studies involving more patients and the parameters related to sarcopenia which should be determined clearly. Since the relationof OA, which is considered to be the most common joint disease, with sarcopenia is emphasized, It is believed that our study will increase awareness in this direction.

\section{ACKNOWLEDGEMENT}

I would like to express my gratitude to Meryem Saraçoğlu, who guided me for her comments, which have helped to improve the paper.

Declaration of conflicting interests :The authors declared no conflicts of interest with respect to the authorship and/or publication of this article.

Funding: The authors received no financial support for the research and/or authorship of this article.

\section{REFERENCES}

1. Felson DT. Epidemiology of hip and knee osteoarthritis. Epidemiologic reviews. 1988;10:1-28.

2. Lawrence RC, Hochberg MC, Kelsey JL, McDuffie FC, Medsger Jr TA, Felts WR, et al. Estimates of the prevalence of selected arthritic and musculoskeletal diseases in the United States. The Journal of rheumatology. 1989;16(4):427-41.

3. Rosenberg IH. Sarcopenia: origins and clinical relevance. The Journal of nutrition. 1997;127(5):990S-1S.

4. Cruz-Jentoft AJ, Baeyens JP, Bauer JM, Boirie Y, Cederholm T, Landi F, et al. Sarcopenia: European consensus on definition and diagnosisReport of the European Working Group on Sarcopenia in Older PeopleA. J. Cruz-Gentoft et al. Age and ageing. 2010;39(4):412-23.

5. Loeser RF. Age-related changes in the musculoskeletal system and the development of osteoarthritis. Clinics in geriatric medicine. 2010;26(3):371-86.

6. Roubenoff R, Heymsfield S, Kehayias J, Cannon JG, Rosenberg I. Standardization of nomenclature of body composition in weight loss. The American journal of clinical nutrition. 1997;66(1):192-6.

7. Scott D, Blizzard L, Fell J, Jones G. Prospective study of self-reported pain, radiographic osteoarthritis, sarcopenia progression, and falls risk in community-dwelling older adults. Arthritis care \& research. 2012;64(1):30-7.

8. Altman R, Asch E, Bloch D, Bole G, Borenstein D, Brandt K, et al. Development of criteria for the classification and reporting of osteoarthritis: classification of osteoarthritis of the knee. Arthritis \& Rheumatism: Official Journal of the American College of Rheumatology. 1986;29(8):1039-49.

9. Demiral Y, Ergor G, Unal B, Semin S, Akvardar Y, Kıvırcık B, et al. Normative data and discriminative properties of short form 36 (SF-36) in Turkish urban population. BMC public health. 2006;6(1):1-8.

10. Rubenstein LZ, Harker JO, Salvà A, Guigoz Y, Vellas B. Screening for undernutrition in geriatric practice: developing the short-form mini-nutritional assessment (MNA-SF). The Journals of Gerontology Series A: Biological Sciences and Medical Sciences. 2001;56(6):M366-M72.

11. Craig CL, Marshall AL, Sjöström M, Bauman AE, Booth ML, Ainsworth BE, et al. International physical activity questionnaire: 12-country reliability and validity. Medicine and science in sports and exercise. 2003;35(8):1381-95.

12. Tatar A, Saltukoglu G. The adaptation of the CES-depression scale into Turkish through the use of confirmatory factor analysis and item response theory and the examination of psychometric characteristics. Klinik Psikofarmakoloji Bülteni-Bulletin of Clinical Psychopharmacology. 2010;20(3):213-27.

13. Podsiadlo D, Richardson S. The timed "Up \& Go": a test of basic functional mobility for frail elderly persons. Journal of the American Geriatrics Society. 1991;39(2):142-8. 
14. McConnell S, Kolopack P, Davis AM. The Western Ontario and McMaster Universities Osteoarthritis Index (WOMAC): a review of its utility and measurement properties. Arthritis Care \& Research: Official Journal of the American College of Rheumatology. 2001;45(5):453-61.

15. Kellgren J, Lawrence J. Radiological assessment of osteo-arthrosis. Annals of the rheumatic diseases. 1957;16(4):494.

16. Kemmler W, Teschler M, Goisser S, Bebenek M, von Stengel S, Bollheimer LC, et al. Prevalence of sarcopenia in Germany and the corresponding effect of osteoarthritis in females 70 years and older living in the community: results of the FORMoSA study. Clinical interventions in aging. 2015;10:1565.

17. Chen Z, Wang Z, Lohman T, Heymsfield SB, Outwater E, Nicholas JS, et al. Dual-energy X-ray absorptiometry is a valid tool for assessing skeletal muscle mass in older women. The journal of nutrition. 2007;137(12):2775-80.

18. Takai Y, Ohta M, Akagi R, Kato E, Wakahara T, Kawakami Y, et al. Applicability of ultrasound muscle thickness measurements for predicting fat-free mass in elderly population. The journal of nutrition, health \& aging. 2014;18(6):579-85.

19. Abe T, Patterson KM, Stover CD, Geddam DA, Tribby AC, Lajza DG, et al. Site-specific thigh muscle loss as an independent phenomenon for age-related muscle loss in middle-aged and older men and women. Age. 2014;36(3):1353-8.

20. Berger J, Bunout D, Barrera G, de la Maza MP, Henriquez S, Leiva L, et al. Rectus femoris (RF) ultrasound for the assessment of muscle mass in older people. Archives of gerontology and geriatrics. 2015;61(1):338.

21. Ismail C, Zabal J, Hernandez HJ, Woletz P, Manning H, Teixeira C, et al. Diagnostic ultrasound estimates of muscle mass and muscle quality discriminate between women with and without sarcopenia. Frontiers in physiology. 2015;6:302.

22. Janssen I, Baumgartner RN, Ross R, Rosenberg IH, Roubenoff R. Skeletal muscle cutpoints associated with elevated physical disability risk in older men and women. American journal of epidemiology. 2004;159(4):413-21.

23. Newman AB, Kupelian V, Visser M, Simonsick EM, Goodpaster BH, Kritchevsky SB, et al. Strength, but not muscle mass, is associated with mortality in the health, aging and body composition study cohort. The Journals of Gerontology Series A: Biological Sciences and Medical Sciences. 2006;61(1):72-7.

24. Lauretani F, Russo CR, Bandinelli S, Bartali B, Cavazzini C, Di Iorio A, et al. Age-associated changes in skeletal muscles and their effect on mobility: an operational diagnosis of sarcopenia. Journal of applied physiology (Bethesda, Md : 1985). 2003;95(5):1851-60.

25. Baumgartner RN, Koehler KM, Gallagher D, Romero L, Heymsfield SB, Ross RR, et al. Epidemiology of sarcopenia among the elderly in New Mexico. American journal of epidemiology. 1998;147(8):755-63.

26. Perez-Sousa MA, Venegas-Sanabria LC, Chavarro-Carvajal DA, Cano-Gutierrez CA, Izquierdo M, Correa-Bautista JE, et al. Gait speed as a mediator of the effect of sarcopenia on dependency in activities of daily living. Journal of cachexia, sarcopenia and muscle. 2019;10(5):1009-15.

27. Strasser EM, Draskovits T, Praschak M, Quittan M, Graf A. Association between ultrasound measurements of muscle thickness, pennation angle, echogenicity and skeletal muscle strength in the elderly. Age. 2013;35(6):2377-88.

28. Toda Y, Segal N, Toda T, Kato A, Toda F. A decline in lower extremity lean body mass per body weight is characteristic of women with early phase osteoarthritis of the knee. The Journal of rheumatology. 2000;27(10):2449-54. 
29. Papalia R, Zampogna B, Torre G, Lanotte A, Vasta S, Albo E, et al. Sarcopenia and its relationship with osteoarthritis: risk factor or direct consequence? Musculoskeletal surgery. 2014;98(1):9-14.

30. Abe T, Yaginuma Y, Fujita E, Thiebaud RS, Kawanishi M, Akamine T. Associations of sit-up ability with sarcopenia classification measures in Japanese older women. Interventional Medicine and Applied Science. $2016 ; 8(4): 152-7$.

31. Choi ES, Cho SH, Kim J-H. Relationship between rectus abdominis muscle thickness and metabolic syndrome in middle-aged men. PloS one. 2017;12(9):e0185040.

32. Kara M, Ekiz T, Kara O, Tiftik T, Malas FU, Demir SO, et al. Does vitamin D affect muscle strength and architecture? An isokinetic and ultrasonographic study. Asia Pacific journal of clinical nutrition. 2017;26(1):85.

33. Kositsawat J, Barry LC, Kuchel GA. C-reactive protein, vitamin D deficiency, and slow gait speed. Journal of the American Geriatrics Society. 2013;61(9):1574-9.

34. Beavers KM, Beavers DP, Houston DK, Harris TB, Hue TF, Koster A, et al. Associations between body composition and gait-speed decline: results from the Health, Aging, and Body Composition study. The American journal of clinical nutrition. 2013;97(3):552-60.

35. Marini E, Buffa R, Saragat B, Coin A, Toffanello ED, Berton L, et al. The potential of classic and specific bioelectrical impedance vector analysis for the assessment of sarcopenia and sarcopenic obesity. Clinical interventions in aging. 2012;7:585.

36. Iannuzzi-Sucich M, Prestwood KM, Kenny AM. Prevalence of sarcopenia and predictors of skeletal muscle mass in healthy, older men and women. The Journals of Gerontology Series A: Biological Sciences and Medical Sciences. 2002;57(12):M772-M7.

37. Rolland Y, Czerwinski S, Abellan Van Kan G, Morley JE, Cesari M, Onder G, et al. Sarcopenia: its assessment, etiology, pathogenesis, consequences and future perspectives. The journal of nutrition, health \& aging. 2008;12(7):433-50.

38. Velazquez Alva Mdel C, Irigoyen Camacho ME, Delgadillo Velazquez J, Lazarevich I. The relationship between sarcopenia,undernutrition, physical mobility and basic activities of daily living in a group of elderly women of Mexico City. Nutricion hospitalaria. 2013;28(2):514-21.

39. Simopoulou T, Malizos K, Iliopoulos D, Stefanou N, Papatheodorou L, Ioannou M, et al. Differential expression of leptin and leptin's receptor isoform (Ob-Rb) mRNA between advanced and minimally affected osteoarthritic cartilage; effect on cartilage metabolism. Osteoarthritis and Cartilage. 2007;15(8):872-83.

40. Sari I, Demir T, Kozaci LD, Akar S, Kavak T, Birlik M, et al. Body composition, insulin, and leptin levels in patients with ankylosing spondylitis. Clinical rheumatology. 2007;26(9):1427-32 .

41. Toussirot E, Streit G, Nguyen NU, Dumoulin G, Le Huede G, Saas P, et al. Adipose tissue, serum adipokines, and ghrelin in patients with ankylosing spondylitis. Metabolism. 2007;56(10):1383-9.

42. Hara K, Horikoshi M, Yamauchi T, Yago H, Miyazaki O, Ebinuma H, et al. Measurement of the highmolecular weight form of adiponectin in plasma is useful for the prediction of insulin resistance and metabolic syndrome. Diabetes care. 2006;29(6):1357-62.

43. Okuno S, Ishimura E, Norimine K, Tsuboniwa N, Kagitani S, Yamakawa K, et al. Serum adiponectin and bone mineral density in male hemodialysis patients. Osteoporosis International. 2012;23(7):2027-35.

44. Hozawa A, Sugawara Y, Tomata Y, Kakizaki M, Tsuboya T, Ohmori-Matsuda K, et al. Relationship between serum adiponectin levels and disability-free survival among community-dwelling elderly individuals: The Tsurugaya Project. The journals of gerontology Series A, Biological sciences and medical sciences. 2012;67(5):530-6. 


\section{Hosted file}

Tables.pdf available at https://authorea.com/users/409535/articles/519162-multi-dimensionalevaluation-of-the-presence-of-sarcopenia-in-patients-with-knee-osteoarthritis 